\title{
A Budget Impact Model of the Addition of Telotristat Ethyl Treatment to the Standard of Care in Patients with Uncontrolled Carcinoid Syndrome
}

\author{
Kelly Fust ${ }^{1} \cdot$ Michael Maschio $^{2} \cdot$ Michele Kohli $^{2} \cdot$ Simron Singh $^{3} \cdot$ D. Mark Pritchard ${ }^{4} \cdot$ Florence Marteau $^{5}$. \\ Peter Myrenfors ${ }^{6} \cdot$ Marion Feuilly $^{5}$
}

Published online: 11 March 2020

(c) The Author(s) 2020

\begin{abstract}
Background Carcinoid syndrome, a rare condition in patients with neuroendocrine tumours, characterised by flushing and diarrhoea, severely affects patients' quality of life. The current carcinoid syndrome standard of care includes somatostatin analogues, but some patients experience uncontrolled symptoms despite somatostatin analogue therapy. Telotristat ethyl is a novel treatment approved by the European Medicines Agency (EMA) and US FDA that significantly reduces bowel movement frequency in patients with uncontrolled carcinoid syndrome.

Objective We developed a model to evaluate the 5-year budget impact of introducing telotristat ethyl to standard care in Swedish patients with uncontrolled carcinoid syndrome.

Methods Treatment response in the 12-week phase III TELESTAR trial (NCT01677910) informed telotristat ethyl efficacy; subsequently, health states were captured by a Markov model using 4-week cycles. TELESTAR open-label extension data informed telotristat ethyl discontinuation. The number of treatment-eligible patients was estimated from literature reviews reporting the prevalence, incidence and mortality of carcinoid syndrome. A Swedish database study informed real-world costs related to carcinoid syndrome and carcinoid heart disease costs. Telotristat ethyl market share was assumed to increase annually from $24 \%$ (year 1) to $70 \%$ (year 5).

Results Over the 5-year model horizon, 44 patients were expected to initiate telotristat ethyl treatment. The cumulative net budget impact of adding telotristat ethyl to current standard of care was $€ 172,346$; per-year costs decreased from $€ 66,495$ (year 1) to $€ 29,818$ (year 5). Increased drug costs from adding telotristat ethyl were offset by reduced costs elsewhere.

Conclusions The expected budget impact of adding telotristat ethyl to the standard of care in Sweden was relatively low, largely because of the rarity of carcinoid syndrome.
\end{abstract}

Electronic supplementary material The online version of this article (https://doi.org/10.1007/s40273-020-00896-5) contains supplementary material, which is available to authorized users.

Marion Feuilly

marion.feuilly@ipsen.com

Optum, 1325 Boylston Street, Boston, MA 02215, USA

2 Formerly affiliated with Optum, 5500 North Service Road, Suite 501, Burlington, ON L7L 6W6, Canada

3 Sunnybrook Research Institute, 2075 Bayview Avenue, Toronto, ON M4N 3M5, Canada

4 Department of Cellular and Molecular Physiology, Institute of Translational Medicine, University of Liverpool, Crown Street, Liverpool L69 3BX, UK

5 Ipsen Pharma, 65 Quai Georges Gorse, 92100 Boulogne-Billancourt, France

6 Ipsen AB, Färögatan 33, 16440 Kista, Sweden

\section{Key Points for Decision Makers}

The expected 5-year budget impact of introducing telotristat ethyl to the standard of care in Swedish patients with uncontrolled carcinoid syndrome was relatively low $(€ 172,346)$, largely because of the rarity of carcinoid syndrome.

Per-year costs decreased from $€ 66,495$ (year 1) to $€ 29,818$ (year 5).

Increased treatment costs from adding telotristat ethyl were offset by reduced costs for other aspects of patient care. 


\section{Introduction}

Carcinoid syndrome (CS) is a rare but serious condition that develops in some patients with neuroendocrine tumours (NETs) [1-3]. Studies from the USA have estimated the prevalence of NETs to range between 0.035 and $0.048 \%$ [4, 5]. Secretion of vasoactive peptides and amines by some NETs, including serotonin, results in the characteristic symptoms of CS, such as cutaneous flushing, diarrhoea and wheezing [1]. CS develops most commonly in patients with small intestinal NETs and hepatic metastases $[3,6]$ and less commonly in patients with pancreatic tumours or lung NETs; CS can also occur when secretions drain directly from tumours into the central circulation [3, 7-9]. Approximately $6-19 \%$ of patients with NETs will develop CS $[1-3,10]$, and 20-50\% of patients with CS develop carcinoid heart disease (CaHD) [11]. In CaHD, secretion of serotonin and other vasoactive substances can cause the deposition of plaques on the right side of the heart, leading to right heart valve dysfunction and possible heart failure [12]. CS progression and response to treatment can be measured through urinary or plasma levels of the serotonin metabolite 5-hydroxyindoleacetic acid (5-HIAA) [13, 14], with elevated urinary 5-HIAA (u5-HIAA) being associated with more severe CS and CaHD $[15,16]$.

The US National Comprehensive Cancer Network guidelines and European Neuroendocrine Tumor Society guidelines currently recommend somatostatin analogues (SSAs), such as lanreotide and octreotide, as treatment for patients with CS $[17,18]$. These long-acting therapeutics can slow tumour progression and, importantly, reduce symptoms of CS [19-23]. In patients with particularly severe CS, symptom burden can be further reduced by dose escalation of long-acting SSAs and the addition of short-acting SSAs and anti-diarrheal therapies to the backbone long-acting SSA therapy [8, 18, 24-29]. However, despite SSA therapy, CS symptoms can persist in approximately $20-40 \%$ of patients [24, 28, 30-32], and over 60\% experience sustained debilitating diarrhoea and flushing [30]. Few options exist for patients whose CS symptoms remain uncontrolled despite treatment with SSAs. The limited alternative treatment options include costly, invasive medical interventions that target tumour load and decrease serotonin secretion [30, 33].

Telotristat ethyl (TE), a novel tryptophan hydroxylase inhibitor, was approved for the treatment of diarrhoea in patients with CS by the European Medicines Agency and US Food and Drug Administration in 2017 and by Health Canada and the Australian Therapeutic Goods Administration in 2018 [34-37]. TE has been shown to reduce daily bowel movement frequency in patients whose CS diarrhoea symptoms are inadequately controlled by SSAs, subsequently improving health-related quality of life when used alongside the current standard of care $[38,39]$. Treatment guidelines in the USA were recently updated to include TE as a treatment option when CS symptoms are poorly controlled [18].

The high healthcare cost of CS, particularly for patients with CS that is uncontrolled by the current standard of care, presents a healthcare, societal, and patient burden. A 2018 Swedish study found the cost per patient with controlled CS over an 8-month period to be $€ 15,500$, rising to $€ 21,700$ per patient with uncontrolled CS [33]. Furthermore, CS symptoms can be debilitating for patients and have a marked impact on health-related quality of life [22, 40-42]; diarrhoea and flushing, in particular, have been associated with reduced physical functioning, increased pain, sleep disturbance, depression and anxiety [41].

TE has been approved for reimbursement by the Swedish Dental and Pharmaceutical Benefits Agency (TLV) [43], but the affordability of using TE in combination with SSA therapy has not yet been demonstrated at the regional level. The aim of this study was to estimate the 5-year budget impact on the Swedish healthcare system of using TE in combination with SSAs, compared with using SSAs only, in patients with inadequately controlled CS. To investigate this, we created a budget impact model, taking into account the drug acquisition cost and all healthcare costs associated with TE treatment.

\section{Methods}

\subsection{Budget Impact Model}

Base-case model analyses were performed from the perspective of the Swedish healthcare system and included direct healthcare costs only. The time horizon of the model analysis was 5 years (2018-2022). This analysis compared two scenarios: (1) a 'world-without TE' scenario in which only SSA therapy was available and (2) a 'world-with TE' scenario in which TE $250 \mathrm{mg}$ was available in addition to SSA therapy. In the model, the size of the target population and the number of patients initiating treatment were estimated. A decision tree and Markov model were then used to estimate the intensity of symptoms and the associated healthcare costs.

\subsubsection{Population and Market Share}

The target population was patients with CS inadequately controlled by SSA therapy. The number of eligible incident cases for years $1-5$ and the number of prevalent cases for year 1 were estimated using data from systematic and targeted literature reviews (Table 1) [4, 5, 26, 44-47]. Figure 1 demonstrates how these data were used to estimate incident and prevalent cases, with the resulting estimates shown in Table 2. For years $2-5$, the number of prevalent cases was 
calculated by the model as the patients continuing from previous years who had not died. Ten incident patients were estimated to be eligible for TE therapy in each year of the analysis (based on incidence estimates in Table 1). Additionally, 72 prevalent cases were eligible for therapy in year 1 . Prevalent patients were assumed to initiate TE treatment in year 1 only.

As SSAs are the current standard of care for CS and for some stages of NETs, all patients were assumed to have received SSA treatment (lanreotide or octreotide) in both the world-with TE and world-without TE scenarios [48]. In the SSA + TE arm, both prevalent and incident cases were considered eligible to commence treatment with TE $250 \mathrm{mg}$ in year 1 (2018), whereas only incident cases were considered eligible in years 2-5 (2019-2022) (Table 2). Based on the assumption that TE has just been introduced and most patients with inadequately controlled disease will receive $\mathrm{TE}$, internal forecasting estimates projected that the market share of TE would increase annually, from $24 \%$ of eligible patients in year 1 to $70 \%$ in year 5 (Table 2). SSA market share did not vary, as TE is to be used in combination with SSAs.

\subsubsection{Decision Tree and Markov Model Structure}

The model structure used to estimate the intensity of symptoms, duration of treatment and associated costs had two components: a decision tree that tracked the response to the initial 12 weeks of TE treatment using data from TELESTAR, and a Markov model using 4-week (28-day) cycles that tracked the longer-term response to treatment for the remaining model time horizon (Fig. 2). Adverse events reported in TELESTAR were not expected to increase healthcare costs significantly or substantially affect patients' quality of life so were not included in the model.

Patients in the 'SSA Only' arm who had a durable response to SSAs during the initial 12 weeks of treatment entered the Markov model in the 'respond to SSA' health state, whereas the remaining patients in the SSA Only arm entered in the 'inadequate response' health state. Patients within the SSA Only arm were assumed to remain on SSA therapies for the entire simulation, regardless of response status. In the SSA + TE arm, patients who achieved a durable response to TE during the initial 12 weeks of treatment entered the Markov model in the 'durable response' health state, whereas patients who did not demonstrate a durable response entered in the 'discontinue TE' health state (and no longer received TE). The inadequate response health state captured patients in the SSA + TE arm who did not maintain a durable response but still continued to receive TE. All health states were stratified by whether the patient had CaHD or not, and the model allowed patients to develop
$\mathrm{CaHD}$ as time progressed. Patients could die in any cycle while in any of the health states.

\subsubsection{Model Inputs}

Initial TE treatment efficacy was informed by data from the 12-week double-blind treatment period of the phase III TELESTAR trial (NCT01677910). A total of 135 patients entered the trial and were randomised to one of three study arms: TE $500 \mathrm{mg}$, TE $250 \mathrm{mg}$, or placebo, each three times daily. All patients remained on the dose of SSA therapy that they were receiving upon entry. Full details of the TELESTAR study design and results have been published previously [38]. For this analyses, a durable response during either stage (the initial 12 weeks of treatment or the 4-week Markov cycles) was defined as a $\geq 30 \%$ reduction in bowel movement frequency for $\geq 50 \%$ of the time, matching the definition used in TELESTAR [38].

The target population for treatment with TE has been defined as patients who have not adequately responded to SSA treatment. In other words, patients who receive TE treatment are not expected to have further symptom reduction with SSAs alone. Therefore, for the base-case analysis, the proportion of durable responders was assumed to be $0 \%$ for patients receiving SSA treatment alone. All patients in the SSA Only arm entered the Markov model in the inadequate response health state and did not ever enter into the respond to SSA health state. Within the SSA + TE treatment arm, the proportion of patients with a durable response attributable to TE after 12 weeks of treatment was $24.4 \%$ (Table 1). This was calculated using data from the TELESTAR trial, in which 20 of the 45 (44.4\%) patients randomised to SSA + TE demonstrated a durable response compared with 9 of the 45 (20\%) patients randomised to SSA + placebo [38].

The 4-week probability of transitioning to the discontinue TE health state was set to 0.0321 based on data from the open-label extension period of TELESTAR, which was not placebo controlled [49]. The probability of transitioning to the inadequate response health state was set to 0 under the assumption that clinicians would discontinue TE treatment if patients stopped showing a durable response to TE. The impact of this assumption was tested in sensitivity analyses by increasing the probability that patients would transition to the inadequate response health state to 0.5 , in the situation where a physician does not discontinue TE in a fragile patient even though the patient is not benefiting from treatment, given a lack of alternative options.

To incorporate $\mathrm{CaHD}$ into the model, the following assumptions were made: an increase in 5-HIAA levels is associated with an increase in CaHD incidence; TE reduces 5-HIAA levels regardless of durable response status [38]; and individuals with $\mathrm{CaHD}$ have an increased risk of death compared with those 
Table 1 Base-case model inputs

\begin{tabular}{|c|c|c|c|c|}
\hline \multicolumn{3}{|l|}{ Target population selection inputs } & \multicolumn{2}{|c|}{ Value [source] } \\
\hline \multicolumn{3}{|l|}{ Total population of Sweden, $n$} & \multicolumn{2}{|c|}{$10,223,505[46]$} \\
\hline \multicolumn{3}{|l|}{ NETs prevalence, $n$ per 100,000 population } & \multicolumn{2}{|c|}{$37[4,44]$} \\
\hline \multicolumn{3}{|l|}{ Annual NETs incidence, $n$ per 100,000 population } & \multicolumn{2}{|c|}{$5.25[5,44]$} \\
\hline \multicolumn{3}{|l|}{ Proportion of patients with intestinal (small bowel) NETs, $\%$} & \multicolumn{2}{|c|}{$17.2[5,44]$} \\
\hline \multicolumn{3}{|l|}{ Proportion of intestinal (small bowel) NETs that are grade $1 / 2, \%$} & \multicolumn{2}{|c|}{$91.0[44,45]$} \\
\hline \multicolumn{3}{|l|}{ Proportion of patients with CS, of those with grade $1 / 2$ intestinal NETs, $\%$} & \multicolumn{2}{|c|}{$30.0[26,44]$} \\
\hline \multicolumn{3}{|l|}{ Proportion of patients with lung NETs, $\%$} & \multicolumn{2}{|c|}{$27.0[5]$} \\
\hline \multicolumn{3}{|l|}{ Proportion of lung NETs that are grade $1 / 2, \%$} & \multicolumn{2}{|c|}{5.4 [45] } \\
\hline \multicolumn{3}{|l|}{ Proportion of patients with CS, of those with grade $1 / 2$ lung NETs, $\%$} & \multicolumn{2}{|c|}{$5.0[47]$} \\
\hline \multicolumn{3}{|l|}{ Proportion of patients with CS that is uncontrolled by SSAs, $\%$} & \multicolumn{2}{|c|}{$40.0^{\mathrm{a}}$} \\
\hline \multicolumn{3}{|l|}{ CaHD inputs $[50]$} & \multicolumn{2}{|r|}{ Value } \\
\hline \multicolumn{3}{|l|}{ Relative mortality associated with $\mathrm{CaHD}$} & \multicolumn{2}{|r|}{2.55} \\
\hline \multicolumn{3}{|l|}{ Baseline incidence of $\mathrm{CaHD}, \%$} & & 2.96 \\
\hline Relative risk for CaHD development in patients with u5-HIAA levels $>300$ & $\mathrm{ol} / 24 \mathrm{~h}$ & & & 2.74 \\
\hline Proportion of TE-treated patients with u5-HIAA levels $<300 \mu \mathrm{mol} / 24 \mathrm{~h}$, ? & & & & 82.0 \\
\hline Proportion of placebo-treated patients with u5-HIAA levels $<300 \mu \mathrm{mol} / 2$ & & & & 55.0 \\
\hline Efficacy inputs for patients receiving $\mathrm{TE}$ & & & & Value \\
\hline Proportion of patients treated with $\mathrm{TE}$ achieving durable response that is att & tted to TE (TELESTAR), \% & & & $24.4^{\mathrm{c}}$ \\
\hline Annual mortality rate for patients with and without $\mathrm{CaHD}$ by year of the an & is [51] & & & \\
\hline Year of analysis & No & & & With CaHD \\
\hline $1(2018)$ & 0.1 & & & 0.3303 \\
\hline $2(2019)$ & 0.1 & & & 0.3375 \\
\hline $3(2020)$ & 0.1 & & & 0.3375 \\
\hline $4(2021)$ & 0.1 & & & 0.3375 \\
\hline $5(2022)$ & 0.1 & & & 0.3375 \\
\hline Medical cost inputs for patients receiving standard of care (SSAs) $[33]^{\mathrm{d}}$ & & & & \\
\hline Item & Controlled CS, $€$ per month & $\begin{array}{l}\text { Uncontrolled CS, } € \text { per } \\
\text { month }\end{array}$ & $\begin{array}{l}\text { Increm } \\
\text { CaHD, }\end{array}$ & $\begin{array}{l}\text { ental cost of } \\
€ \text { per month }\end{array}$ \\
\hline Medical and surgical interventions & 143.60 & 494.00 & 170.92 & \\
\hline Examinations and imaging & 78.78 & 264.75 & 87.58 & \\
\hline Other outpatient visits & 57.84 & 74.75 & 2.08 & \\
\hline Inpatient admissions & 260.13 & 263.75 & 32.75 & \\
\hline SSAs & 1257.88 & 1521.50 & 220.50 & \\
\hline IFN- $\alpha$ & 137.63 & 83.88 & - & \\
\hline Chemotherapy & 0.94 & 5.60 & - & \\
\hline Other diarrhoea therapy & 1.98 & 4.11 & - & \\
\hline CaHD drug costs & - & - & 1.83 & \\
\hline Sum of monthly medical cost inputs for patients receiving standard of care & 1938.78 & 2712.34 & 515.66 & \\
\hline Drug costs for patients receiving $\mathrm{SSAs}+\mathrm{TE}^{\mathrm{f}}$ & & & & $€$ per 4 weeks \\
\hline TE & & & & 982.27 \\
\hline
\end{tabular}

CaHD carcinoid heart disease, $C S$ carcinoid syndrome, IFN interferon, NET neuroendocrine tumour, SSA somatostatin analogue, TE telotristat ethyl, $u 5$-HIAA urinary 5-hydroxyindoleacetic acid

${ }^{\text {a}}$ Forecasting assumption based on interviews with healthcare professionals

${ }^{\mathrm{b}}$ Based on a post-hoc analysis of TELESTAR clinical trial data

${ }^{\mathrm{c}}$ Data derived from Kulke et al. [38], in which 20 of 45 (44.4\%) patients randomised to SSA + TE demonstrated a durable response, compared with 9 of $45(20 \%)$ patients randomised to SSA + placebo; therefore, for the purpose of this model, $24.4 \%$ of the response was presumed to be attributable to TE

${ }^{\mathrm{d}}$ Values from Lesén et al. [33], which cover an 8-month period, were divided by eight to determine per-month costs

${ }^{\mathrm{e}}$ Annual CaHD incidence was assumed to be $2.96 \%$ for patients with 5 -HIAA $<300 \mathrm{mmol} / 24 \mathrm{~h}$ [50]

${ }^{\mathrm{f}}$ TE not included in standard of care 


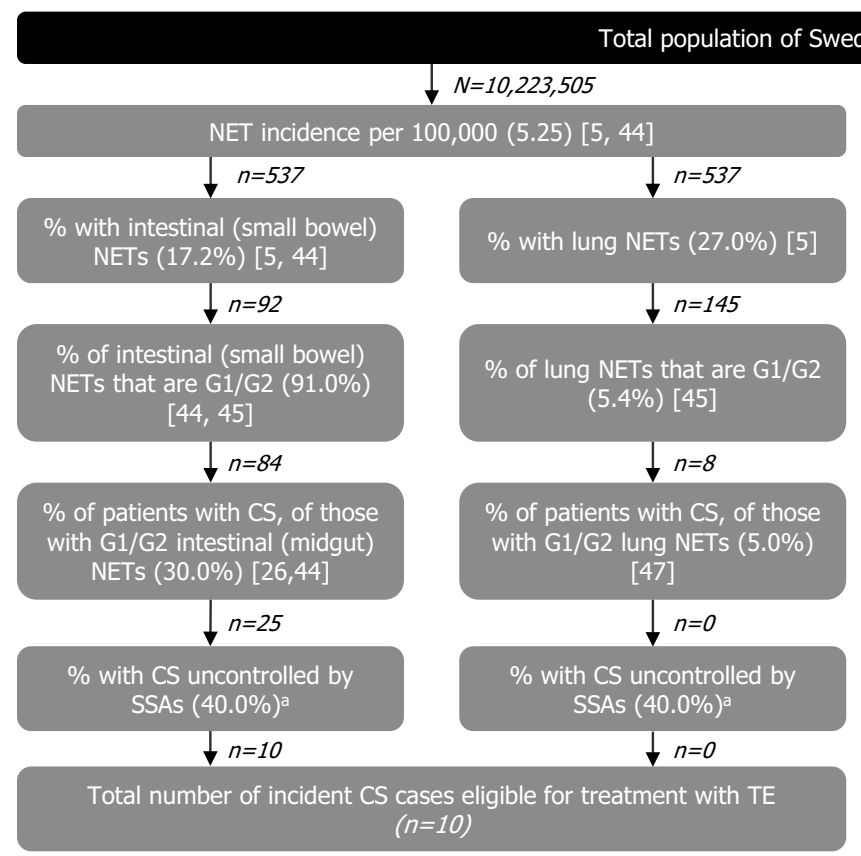

Fig. 1 Selection criteria for determining the target population (incident and prevalent CS cases eligible for treatment with TE), based on data from systematic and targeted literature reviews. Data from literature reviews were used to estimate the target population selection inputs as listed in Table 1. Resulting estimates used in the model are

\section{$N=10,223,505$}

NET prevalence per 100,000 , Year 1 only $(37)[4,44]$

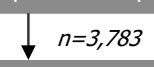

$\%$ with intestinal (small bowel) NETS $(17.2 \%)[5,44]$

$$
\downarrow n=651
$$

$\%$ of intestinal (small bowel)

NETs that are G1/G2 $(91.0 \%)$ $[44,45]$

$n=592$

$\%$ of patients with CS, of those

with $\mathrm{G} 1 / \mathrm{G} 2$ intestinal (midgut)

NETs $(30.0 \%)[26,44]$

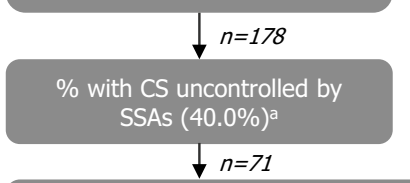

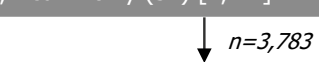

$\%$ with lung NETs (27.0\%) [5]

$n=1,02$

$\%$ of lung NETs that are $\mathrm{G} 1 / \mathrm{G} 2$ $(5.4 \%)[45]$

$n=55$

$\%$ of patients with CS, of those

with G1/G2 lung NETs (5.0\%) [47]

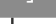

$n=3$

$\%$ with CS uncontrolled by $\operatorname{SSAS}(40.0 \%)^{2}$

$\downarrow n=1$
Total number of prevalent CS cases eligible for treatment with TE $(n=72)$

shown in (parentheses), with the relevant sources in [brackets]. ${ }^{\text {aFore- }}$ casting assumption based on interviews with healthcare professionals. $C S$ carcinoid syndrome, G1/G2 grade 1/grade 2, NET neuroendocrine tumour, $S S A$ somatostatin analogue, $T E$ telotristat ethyl
Table 2 Forecast base-case eligible population and market share assumptions for the world-without TE and worldwith TE scenarios over 5 years

\begin{tabular}{|c|c|c|c|c|c|}
\hline & Year 1 (2018) & Year 2 (2019) & Year $3(2020)$ & Year $4(2021)$ & Year $5(2022)$ \\
\hline \multicolumn{6}{|l|}{ Population } \\
\hline Prevalent cases & 72 & 74 & 71 & 68 & 66 \\
\hline Incident cases & 10 & 10 & 10 & 10 & 10 \\
\hline Total cases ${ }^{\mathrm{a}}$ & 82 & 84 & 81 & $79^{\mathrm{b}}$ & 76 \\
\hline \multicolumn{6}{|l|}{ Market share } \\
\hline \multicolumn{6}{|c|}{ World-without TE scenario } \\
\hline SSAs only, $\%$ & 100 & 100 & 100 & 100 & 100 \\
\hline \multicolumn{6}{|c|}{ World-with TE scenario } \\
\hline SSA + TEs, $\%$ & 24 & 52 & 58 & 60 & 70 \\
\hline SSAs only, \% & 76 & 48 & 42 & 40 & 30 \\
\hline
\end{tabular}

$C S$ carcinoid syndrome, SSA somatostatin analogue, $T E$ telotristat ethyl

Prevalence and incidence of uncontrolled CS was assumed to be the same for scenarios both with and without TE; values have been rounded to the nearest whole number

${ }^{\text {a }}$ Total cases decreases from year 3 as the rate of mortality for patients with prevalent CS is higher than the incidence of new patients, based on mortality estimates in Table 1

${ }^{\mathrm{b}}$ Prevalent and incident cases do not sum to 78 due to rounding without. Levels of u5-HIAA were informed through analysis of data from the TELESTAR clinical trial [38]. The incidence of CaHD based on u5-HIAA was calculated using CaHD incidence as reported in Bhattacharyya et al. [50], which was the only study identified in a systematic literature review examining the link between 5-HIAA levels and CaHD incidence (Table 1).
The mean age of the cohort at the start of the simulation was assumed to be 64 years based on TELESTAR patient characteristics [38]. Relative 5-year survival rates for grade 1 and 2 tumours located in the intestinal tract were determined through application of the relative survival rates in Korse et al. [45] to the Swedish age-specific mortality rates. 
Fig. 2 Markov model for treatment response after the initial 12-week treatment period. Patients with a durable response to TE begin the Markov process in the 'SSA + TE' arm. Patients who do not experience a durable response are assumed to discontinue TE but maintain SSA treatment and start in the 'SSA only' arm. The transition between durable response and inadequate response in the $\mathrm{SSA}+\mathrm{TE}$ arm was set to 0 for the base-case analysis. There were no deaths during the first 12 weeks of the model (the trial period). $C a H D$ carcinoid heart disease, SSA somatostatin analogue, $T E$ telotristat ethyl

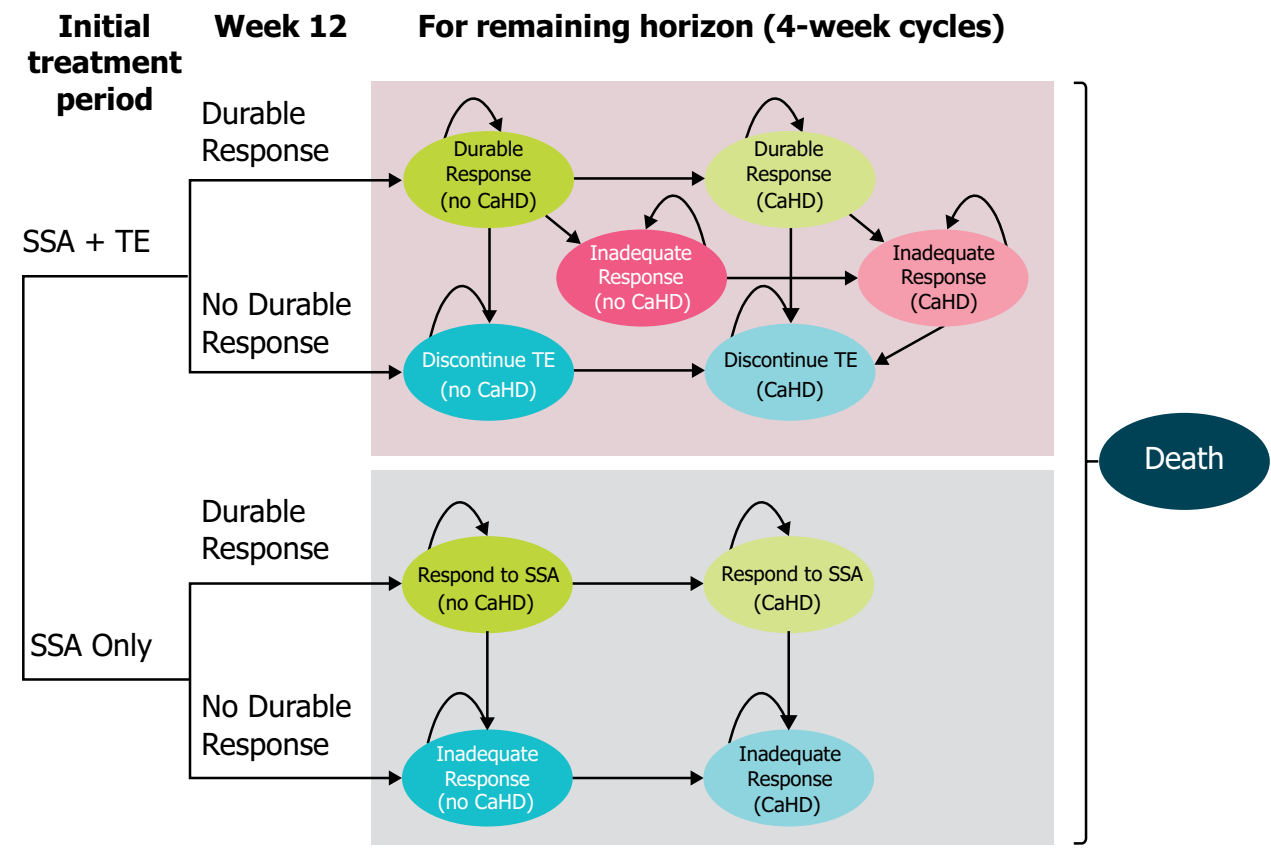

For the base case, the mortality risk associated with CaHD was calculated using data from Westberg et al. [51]. Basecase model inputs for mortality risk are shown in Table 1.

\subsubsection{Cost Inputs}

The unit cost of TE in Swedish krona (SEK) $(10,100.00)$ [43] was converted to $€$ using the European Central Bank conversion rate as of 28 November $2018(€ 1=$ SEK10.2823). Therefore, the unit cost of TE in Sweden is approximately $€ 11.69$ per $250 \mathrm{mg}$ pill, cumulating in a total cost of $€ 982.27$ over 4 weeks (three pills per day). Patients in the SSA + TE arm during the initial treatment period and those in the durable response and inadequate response to TE health states within the Markov model incurred this cost.

Real-world CS and CaHD-related costs, including healthcare resource use (frequency per month), drug acquisition and SSA dosage (cost of average dose) for patients with controlled and uncontrolled CS were obtained from a retrospective Swedish database study [33] (Table 1). A number of symptoms not limited to diarrhoea may contribute to cost differences between responders and non-responders in the model. The costs associated with patients with controlled CS were assigned to the respond to SSA and durable response to TE health states. The costs associated with patients with uncontrolled CS were assigned to all remaining non-responder health states. Incremental costs for CaHD were determined by calculating the difference in monthly costs between patients with and without CaHD. The cost year for all model inputs was 2015, except for the Swedish total population size and TE unit cost, which used actual values as of reimbursement approval by the TLV in 2018 .

\subsection{Deterministic Sensitivity Analyses}

Deterministic (one-way) sensitivity analyses were conducted to assess the impact of model parameters. Model parameters were varied using $95 \%$ confidence intervals (CIs) or plausible ranges as reported in the published literature. When the published study did not report $95 \%$ CIs or ranges, plausible ranges were specified or informed by clinical expert opinion, obtained through interviews with experts identified as knowledgeable about NET management and economic models. Ranges for inputs included in the sensitivity analyses are shown in electronic supplementary material 1.

\section{Results}

\subsection{Base-Case Analysis}

\subsubsection{Number of Patients Treated}

Based on the assumed TE market share and number of CS cases in the base population, approximately 44 patients would initiate TE therapy during the 5-year time horizon (Table 3). Because prevalent patients were assumed to initiate TE treatment in year 1 only, the number of patients expected to begin TE therapy was highest in year 1 (19.8).

\subsubsection{Costs}

Table 4 shows a full breakdown of costs over 5 years in the world-without TE and world-with TE scenarios. The total cost in year 1 was $€ 2,793,111$ in the world-without TE 
scenario and $€ 2,859,606$ in the world-with TE scenario and subsequently declined over the 5 -year period. The worldwith TE scenario resulted in total drug costs $€ 269,056$ higher than in the world-without TE scenario over the 5-year period. Approximately $40 \%$ was offset by the reduced costs for other aspects of patient care in the world-with TE scenario, including medical and surgical interventions, examinations and imaging, outpatient visits, inpatient admissions, chemotherapy, other diarrhoea therapy and CaHD drug costs (Table 4).

The cumulative 5-year net budget impact of introducing TE to the current standard of care was $€ 172,346$; the cumulative cost over 5 years of treatment was $€ 13,263,595$ for the world-without TE scenario and $€ 13,435,941$ for the world-with TE scenario (Table 5). The net budget impact per year of introducing TE to the current standard of care was predicted to decrease from $€ 66,495$ in year 1 to $€ 29,818$ in year 5 .

\subsection{Deterministic Sensitivity Analyses}

Results of the deterministic sensitivity analysis are shown in Fig. 3. The 5-year net budget impact of TE was most sensitive to the proportion of patients who continued TE therapy after 12 weeks of treatment despite lack of durable response. If $50 \%$ of patients who did not achieve a durable response continued TE therapy, the cumulative budget impact would increase to $€ 696,661$. The price of TE $(250 \mathrm{mg})$ was the second biggest factor affecting the model outcomes, with a $25 \%$ increase or decrease resulting in a 5-year cumulative net budget impact of $€ 251,668$ and $€ 92,824$, respectively.

\section{Discussion}

Despite most patients with NETs receiving SSA therapy, many of those with CS continue to experience uncontrolled symptoms [24, 28, 30, 31, 52]. TE offers an effective, novel treatment option for patients with CS with uncontrolled diarrhoea. Used in combination with SSAs, TE has been shown to offer effective relief from CS diarrhoea and to improve the quality of life in patients who experience a durable response to treatment [38]. This study investigated the budget impact of the addition of TE to the current standard of care for CS in Sweden.

The total cost per year of treatment for patients with CS inclusive of TE therapy in Sweden declined over the 5-year time-period, from $€ 2,859,606$ in year 1 to $€ 2,523,961$ in year 5 , as patients were assumed to discontinue TE due to adverse events, lack of effectiveness and CS progression. The cumulative 5-year net budget impact resulting from the addition of TE was relatively low at $€ 172,346$, with a per-year net cost of $€ 66,495$ in year 1 and decreasing thereafter.

The relatively low budget impact of adding TE to the existing standard of care in Sweden is likely due to two reasons. First, CS is a component of a rare disease, and the number of total prevalent and incident patients eligible for TE was estimated at approximately 80 patients each year over the 5 years; according to market share assumptions, only 44 patients would initiate treatment with TE by year 5. This aligns with findings from a 2014 study assessing the budget impact of orphan medicinal products in Sweden and France, in which the low overall number of annual sales made the costs associated with these treatments sustainable [53]. Further to this, the TE budget impact model assumed that patients who responded to TE would gradually discontinue treatment either as the disease progressed, TE
Table 3 Number of patients initiating TE treatment in the world-with TE scenario over 5 years

\begin{tabular}{llllll}
\hline & Year 1 (2018) & Year 2 (2019) & Year 3 (2020) & Year 4 (2021) & Year 5 (2022) \\
\hline Prevalent cases & $17.3^{\mathrm{a}}$ & 0.0 & 0.0 & 0.0 & 0.0 \\
Incident cases & 2.5 & 5.3 & 5.9 & 6.1 & 7.2 \\
$\quad$ Total cases & 19.8 & 5.3 & 5.9 & 6.1 & 7.2 \\
$\quad \begin{array}{l}\text { Number of patients } \\
\quad 14.9\end{array}$ & 4.0 & 4.5 & 4.6 & 5.4 \\
$\quad \begin{array}{l}\text { discontinuing TE after } \\
\text { initial 12-week treat- }\end{array}$ & & & & & \\
$\quad$ ment period & & & & 1.7 \\
$\begin{array}{l}\text { Number of patients con- } \\
\quad \text { tinuing TE after initial }\end{array}$ & 4.8 & 1.3 & 1.4 & & \\
$\quad \begin{array}{l}\text { 12-week treatment } \\
\text { period }\end{array}$ & & & & & \\
\hline
\end{tabular}

Numbers may not sum due to rounding

$T E$ telotristat ethyl

${ }^{\mathrm{a}} \mathrm{TE}$ was granted marketing authorisation in the EU prior to the start of year 1; therefore, prevalent patients were assumed to have had an opportunity to switch to TE in year 1 but were not expected to switch in following years 
Table 4 Total costs over 5 years from the world-without TE and world-with TE scenarios

\begin{tabular}{|c|c|c|c|c|c|c|}
\hline & Year 1 (2018) & Year 2 (2019) & Year 3 (2020) & Year $4(2021)$ & Year 5 (2022) & Total \\
\hline \multicolumn{7}{|l|}{ World-without TE scenario } \\
\hline Total costs, $€$ & $2,793,111$ & $2,742,376$ & $2,659,435$ & $2,574,530$ & $2,494,143$ & $13,263,595$ \\
\hline SSA + TE drug costs & $1,565,879$ & $1,534,877$ & $1,486,779$ & $1,438,271$ & $1,392,723$ & $7,418,528$ \\
\hline TE $250 \mathrm{mg}$ & - & - & - & - & - & - \\
\hline SSA only & $1,565,879$ & $1,534,877$ & $1,486,779$ & $1,438,271$ & $1,392,723$ & $7,418,528$ \\
\hline SSA component of TE arm & - & - & - & - & - & - \\
\hline Medical and surgical intervention & 509,755 & 503,360 & 490,013 & 475,535 & 461,403 & $2,440,068$ \\
\hline Examinations and imaging & 273,139 & 269,564 & 262,319 & 254,508 & 246,908 & $1,306,438$ \\
\hline Other outpatient visits & 76,812 & 74,965 & 72,402 & 69,907 & 67,612 & 361,698 \\
\hline Inpatient admissions & 271,369 & 265,792 & 257,330 & 248,851 & 240,919 & $1,284,260$ \\
\hline IFN- $\alpha$ & 86,157 & 83,998 & 81,069 & 78,239 & 75,648 & 405,112 \\
\hline Chemotherapy & 5752 & 5608 & 5413 & 5224 & 5051 & 27,048 \\
\hline Other diarrhoea therapy & 4224 & 4119 & 3975 & 3836 & 3709 & 19,863 \\
\hline $\mathrm{CaHD}$ drug cost & 25 & 93 & 134 & 158 & 170 & 580 \\
\hline \multicolumn{7}{|l|}{ World-with TE scenario } \\
\hline Total costs, $€$ & $2,859,606$ & $2,766,700$ & $2,684,974$ & $2,600,700$ & $2,523,961$ & $13,435,941$ \\
\hline $\mathrm{SSA}+\mathrm{TE}$ drug costs & $1,652,700$ & $1,580,473$ & $1,531,252$ & $1,482,313$ & $1,440,846$ & $7,687,584$ \\
\hline TE $250 \mathrm{mg}$ & 97,334 & 56,542 & 54,083 & 52,965 & 57,136 & 318,061 \\
\hline SSA only & $1,190,068$ & $1,112,025$ & $1,017,163$ & 927,248 & 828,840 & $5,075,343$ \\
\hline SSA component of TE arm & 365,299 & 411,906 & 460,005 & 502,101 & 554,870 & $2,294,180$ \\
\hline Medical and surgical intervention & 495,791 & 488,730 & 476,947 & 463,149 & 448,672 & $2,373,289$ \\
\hline Examinations and imaging & 265,728 & 261,801 & 255,387 & 247,938 & 240,155 & $1,271,009$ \\
\hline Other outpatient visits & 76,139 & 74,268 & 71,790 & 69,336 & 67,032 & 358,566 \\
\hline Inpatient admissions & 271,221 & 265,648 & 257,235 & 248,802 & 240,904 & $1,283,811$ \\
\hline IFN- $\alpha$ & 88,295 & 86,243 & 83,093 & 80,182 & 77,667 & 415,480 \\
\hline Chemotherapy & 5567 & 5415 & 5241 & 5061 & 4883 & 26,167 \\
\hline Other diarrhoea therapy & 4139 & 4030 & 3896 & 3762 & 3633 & 19,461 \\
\hline CaHD drug cost & 24 & 91 & 133 & 156 & 168 & 574 \\
\hline
\end{tabular}

$C a H D$ carcinoid heart disease, IFN interferon, SSA somatostatin analogue, TE telotristat ethyl

Table 5 Costs and net budget impact of TE for the worldwithout TE and world-with TE scenarios

\begin{tabular}{|c|c|c|c|c|c|c|}
\hline & Year $1(2018)$ & Year 2 (2019) & Year 3 (2020) & Year $4(2021)$ & Year 5 (2022) & Total \\
\hline \multicolumn{7}{|c|}{ World-without TE scenario } \\
\hline Total costs, $€$ & $2,793,111$ & $2,742,376$ & $2,659,435$ & $2,574,530$ & $2,494,143$ & $13,263,595$ \\
\hline PMPM, € & 0.02 & 0.02 & 0.02 & 0.02 & 0.02 & - \\
\hline PPPM, € & 2825 & 2723 & 2727 & 2729 & 2731 & - \\
\hline \multicolumn{7}{|c|}{ World-with TE scenario } \\
\hline Total costs, $€$ & $2,859,606$ & $2,766,700$ & $2,684,974$ & $2,600,700$ & $2,523,961$ & $13,435,941$ \\
\hline PMPM, € & 0.02 & 0.02 & 0.02 & 0.02 & 0.02 & - \\
\hline PPPM, € & 2892 & 2747 & 2752 & 2756 & 2763 & - \\
\hline \multicolumn{7}{|c|}{ Net budget impact of TE } \\
\hline Total costs, $€$ & 66,495 & 24,323 & 25,539 & 26,171 & 29,818 & 172,346 \\
\hline PMPM, $€$ & 0.00 & 0.00 & 0.00 & 0.00 & 0.00 & - \\
\hline PPPM, € & 67.26 & 24.06 & 25.74 & 26.85 & 31.29 & - \\
\hline
\end{tabular}

$P M P M$ per population member per month, $P P P M$ per patient per month, $T E$ telotristat ethyl 
HRU medical and surgical interventions, number/month, uncontrolled CS

$\%$ with intestinal (small bowel) NETs

$\%$ with CS and grade $1 / 2$ intestinal (midgut) NETs

HRU SSAs, number/month, uncontrolled CS

HRU inpatient admissions, number/month, uncontrolled CS

HRU SSAs, number/month, controlled CS

HRU inpatient admissions, number/month, controlled CS

\section{$\%$ failure to discontinue TE despite lack of efficacy ${ }^{a}$ Price of TE dose $(€)^{\mathrm{b}}$ \\ $\%$ with CS inadequately controlled by SSAS}

- Lower estimates for value Upper estimates for value

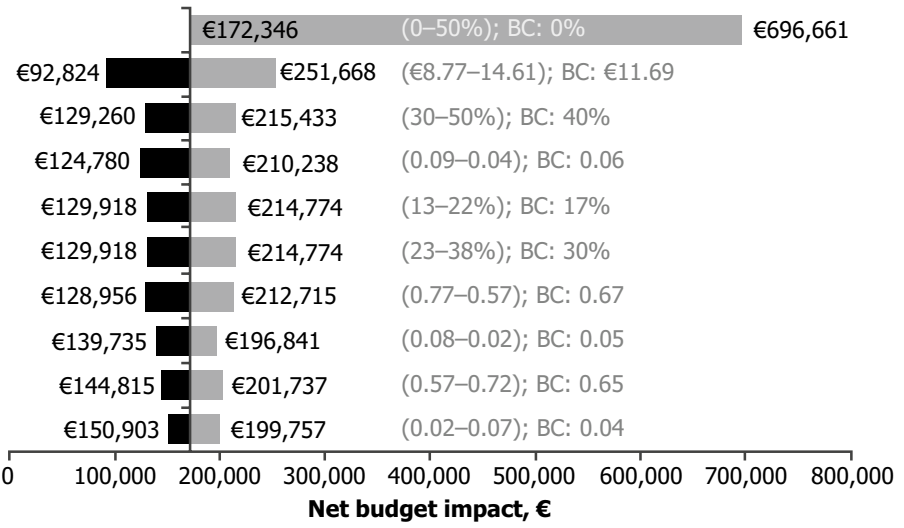

eters for the sensitivity analyses. $B C$ base case, $C S$ carcinoid syndrome, $H R U$ healthcare resource use, NET neuroendocrine tumour, $S S A$ somatostatin analogue, TE telotristat ethyl TE treatment using data from TELESTAR. ${ }^{\mathrm{b}}$ Parameter varied based on arbitrary ranges. Values in parentheses are the variation param-

effectiveness decreased, adverse events occurred or patients died, which would also contribute to the small economic impact.

Second, the improved diarrhoea symptom control resulting from treatment with TE translated into overall healthcare system cost savings that offset the pharmacy cost of TE, an outcome previously reported for other treatments in rare chronic diseases [54, 55]. Although patients who respond to TE will still require ongoing monitoring for tumour progression, they would avoid the increased costs related to uncontrolled CS. The total costs per patient per year associated with medical and surgical interventions, examinations and imaging, outpatient visits, inpatient admissions, chemotherapy and other diarrhoea therapies were lower in the world-with TE scenario. This is in accordance with other studies showing that patients with CS who experience flushing and diarrhoea symptoms incur around \$US14,766-29,890 more per year in healthcare costs than those experiencing improvements in symptoms $[10,56]$.

Our results are consistent with those from a US study that also showed a minimal budget impact on a US health plan of adding TE to SSA therapy [57]. In this study, the budget impact of adding TE totalled \$US687,330 over 3 years. With a similar assumed market share (28\% in year $1,42 \%$ in year 2 , and $55 \%$ in year 3 ), the net annual overall healthcare cost of TE (\$US55-109 million) was well under the Institute for Clinical and Economic Review (ICER) threshold for new molecular entities (\$US915 million per year) when the study was published in 2017 [57]. While the ICER decreased the threshold to \$US819 million in May 2019, the budget impact of adding TE to the standard of care in the USA still falls well under this amount [58].
A limitation of our study was the lack of readily available data, which is often a challenge when conducting budget impact analyses of treatments for orphan diseases [59, 60]. For example, prevalence and incidence data specific to Sweden were not available, so this analysis used data from a systematic review conducted for Europe and the USA to estimate the size of the target population [44]. The derivation of cost inputs for patients with controlled and uncontrolled CS from Swedish databases presented another limitation for this study. It was assumed that patients demonstrating a durable response to TE would have the same resource use as patients with controlled CS, and patients without a durable response to TE would have that of patients with uncontrolled CS (despite the fact that the definition of a durable response relates to reductions in symptoms of diarrhoea). The impact of this assumption on our budget impact estimate is unknown. However, because diarrhoea is the most burdensome symptom of CS, this model focused on cost outcomes associated with this symptom, though durable responders have also been shown to have improvements in other symptoms that would not be captured by this model [39]. It should be noted that this model does not account for the lost productivity to individual patients and society in general from the impact of CS on quality of life and consequences such as missed workdays.

\section{Conclusions}

Treatment with TE alongside SSAs more often results in durable improvement of diarrhoea symptoms than use of SSAs alone; symptomatic relief can lead to improved quality of life for patients with NETs and CS. This budget impact model demonstrated that TE could be an affordable addition 
to the current standard of care for patients with NETs and CS in Sweden. Therefore, further research assessing the health and budget impacts of TE and other treatments for CS and NETs in Sweden would be beneficial and is warranted.

Acknowledgements The authors thank the Optum team for model development, specifically Laura Pastor, formerly of Optum, Burlington, Ontario, Canada, for model input estimation and quality assurance. The authors also thank Milton Weinstein of Harvard T.H. Chan School of Public Health, Boston, Massachusetts, USA, for review of and feedback on the overall model design, and Anna Jonasson of Ipsen Pharma, Kista, Sweden, and Staffan Welin, MD, PhD of Uppsala Universitet, Uppsala, Sweden for model input estimation. The authors acknowledge Amelia Frizell-Armitage, $\mathrm{PhD}$, and Kristian Clausen, MPH of Costello Medical, Cambridge, UK, for providing medical writing and editorial support, which was funded by Ipsen Pharma (Boulogne-Billancourt, France) in accordance with Good Publication Practice guidelines. This study and model development was sponsored by Ipsen Pharma (Boulogne-Billancourt, France).

\section{Compliance with Ethical Standards}

Funding Medical writing and editorial support was funded by Ipsen Pharma (Boulogne-Billancourt, France) in accordance with good publication practice guidelines. This study and model development was sponsored by Ipsen Pharma (Boulogne-Billancourt, France).

Conflict of interest KF is employed by Optum. MM and MK were formerly employed by Optum. SS has received honoraria from Ipsen Pharma and Novartis, and research funding/fellowship support from Ipsen Pharma, Novartis, Merck EMD Serono and Pfizer. DMP has received honoraria from Advanced Accelerator Applications (AAA) and Ipsen Pharma, and research funding from Trio Medicines Ltd. FM, PM, MF are employed by Ipsen Pharma.

Data Sharing Where patient data can be anonymised, Ipsen will share all individual participant data that underlie the results reported in this article with qualified researchers who provide a valid research question. Study documents, such as the study protocol and clinical study report, are not always available. Proposals should be submitted to DataSharing@Ipsen.com and will be assessed by a scientific review board. Data are available beginning 6 months and ending 5 years after publication; after this time, only raw data may be available.

Author Contributions KF, MM, MK, SS, DMP, FM and MF made substantial contributions to the study conception/design or acquisition/ analysis/interpretation of the data. KF, MM, MK, SS, DMP, FM, PM and MF drafted the publication or revised it critically for important intellectual content. MM, MK, SS, DMP, FM, PM and MF approved the manuscript for publication.

Open Access This article is licensed under a Creative Commons Attribution-NonCommercial 4.0 International License, which permits any non-commercial use, sharing, adaptation, distribution and reproduction in any medium or format, as long as you give appropriate credit to the original author(s) and the source, provide a link to the Creative Commons licence, and indicate if changes were made. The images or other third party material in this article are included in the article's Creative Commons licence, unless indicated otherwise in a credit line to the material. If material is not included in the article's Creative Commons licence and your intended use is not permitted by statutory regulation or exceeds the permitted use, you will need to obtain permission directly from the copyright holder.To view a copy of this licence, visit http://creativecommons.org/licenses/by-nc/4.0/.

\section{References}

1. Halperin DM, Shen C, Dasari A, Xu Y, Chu Y, Zhou S, et al. Frequency of carcinoid syndrome at neuroendocrine tumour diagnosis: a population-based study. Lancet Oncol. 2017;18(4):525-34.

2. Soga J. Carcinoids and their variant endocrinomas. An analysis of 11842 reported cases. J Exp Clin Cancer Res. 2003;22(4):517-30.

3. Tomassetti P, Migliori M, Lalli S, Campana D, Tomassetti V, Corinaldesi R. Epidemiology, clinical features and diagnosis of gastroenteropancreatic endocrine tumours. Ann Oncol. 2001;12(Suppl 2):S95-9.

4. Dasari A, Shen C, Halperin D, Zhao B, Zhou S, Xu Y, et al. Trends in the incidence, prevalence, and survival outcomes in patients with neuroendocrine tumors in the United States. JAMA Oncol. 2017;3(10):1335-42.

5. Yao JC, Hassan M, Phan A, Dagohoy C, Leary C, Mares JE, et al. One hundred years after "carcinoid": epidemiology of and prognostic factors for neuroendocrine tumors in 35,825 cases in the United States. J Clin Oncol. 2008;26(18):3063-72.

6. Kaltsas GA, Besser GM, Grossman AB. The diagnosis and medical management of advanced neuroendocrine tumors. Endocr Rev. 2004;25(3):458-511.

7. Papadogias D, Makras P, Kossivakis K, Kontogeorgos G, Piaditis G, Kaltsas G. Carcinoid syndrome and carcinoid crisis secondary to a metastatic carcinoid tumour of the lung: a therapeutic challenge. Eur J Gastroenterol Hepatol. 2007;19(12):1154-9.

8. Jensen RT, Cadiot G, Brandi ML, de Herder WW, Kaltsas G, Komminoth P, et al. ENETS consensus guidelines for the management of patients with digestive neuroendocrine neoplasms: functional pancreatic endocrine tumor syndromes. Neuroendocrinology. 2012;95(2):98-119.

9. Stamatakos M, Kontzoglou K, Sargedi C, Tsaknaki S, Iannescu $\mathrm{R}$, Safioleas C, et al. Gastrointestinal carcinoid tumors: diagnosis and treatment. Chirurgia (Bucur). 2010;105(6):759-66.

10. Broder MS, Chang E, Romanus D, Cherepanov D, Neary MP. Healthcare and economic impact of diarrhea in patients with carcinoid syndrome. World J Gastroenterol. 2016;22(6):2118-25.

11. Davar J, Connolly HM, Caplin ME, Pavel M, Zacks J, Bhattacharyya $S$, et al. Diagnosing and managing carcinoid heart disease in patients with neuroendocrine tumors: an expert statement. J Am Coll Cardiol. 2017;69(10):1288-304.

12. Grozinsky-Glasberg S, Grossman AB, Gross DJ. Carcinoid heart disease: from pathophysiology to treatment-'something in the way it moves'. Neuroendocrinology. 2015;101(4):263-73.

13. Boudreaux JP, Klimstra DS, Hassan MM, Woltering EA, Jensen RT, Goldsmith SJ, et al. The NANETS consensus guideline for the diagnosis and management of neuroendocrine tumors: well-differentiated neuroendocrine tumors of the jejunum, ileum, appendix, and cecum. Pancreas. 2010;39(6):753-66.

14. Liu Q, Yang Q, Sun W, Vogel P, Heydorn W, Yu XQ, et al. Discovery and characterization of novel tryptophan hydroxylase inhibitors that selectively inhibit serotonin synthesis in the gastrointestinal tract. J Pharmacol Exp Ther. 2008;325(1):47-55.

15. Fox DJ, Khattar RS. Carcinoid heart disease: presentation, diagnosis, and management. Heart. 2004;90(10):1224-8.

16. Kulke MH, Benson AB, Bergsland E, Berlin JD, Blaszkowsky LS, Choti MA, et al. Neuroendocrine tumors. J Natl Compr Cancer Netw. 2012;10(6):724-64.

17. Delle Fave G, O'Toole D, Sundin A, Taal B, Ferolla P, Ramage JK, et al. ENETS consensus guidelines update for 
gastroduodenal neuroendocrine neoplasms. Neuroendocrinology. 2016;103(2):119-24.

18. National Comprehensive Cancer Network. Neuroendocrine and adrenal tumors. NCCN clinical practice guidelines in oncology. https://www.nccn.org/professionals/physician_gls/pdf/neuroendoc rine.pdf. Accessed 21 March 2019.

19. Caplin ME, Pavel M, Cwikla JB, Phan AT, Raderer M, Sedlackova $\mathrm{E}$, et al. Lanreotide in metastatic enteropancreatic neuroendocrine tumors. N Engl J Med. 2014;371(3):224-33.

20. Rinke A, Muller HH, Schade-Brittinger C, Klose KJ, Barth P, Wied M, et al. Placebo-controlled, double-blind, prospective, randomized study on the effect of octreotide LAR in the control of tumor growth in patients with metastatic neuroendocrine midgut tumors: a report from the PROMID study group. J Clin Oncol. 2009;27(28):4656-63.

21. Vinik AI, Wolin EM, Liyanage N, Gomez-Panzani E, Fisher GA. Evaluation of lanreotide depot/autogel efficacy and safety as a carcinoid syndrome treatment (elect): a randomized, double-blind, placebo-controlled trial. Endocr Pract. 2016;22(9):1068-80.

22. Ruszniewski P, Valle JW, Lombard-Bohas C, Cuthbertson DJ, Perros $\mathrm{P}$, Holubec L, et al. Patient-reported outcomes with lanreotide autogel/depot for carcinoid syndrome: an international observational study. Dig Liver Dis. 2016;48(5):552-8.

23. Harris AG, Redfern JS. Octreotide treatment of carcinoid syndrome: analysis of published dose-titration data. Aliment Pharmacol Ther. 1995;9(4):387-94.

24. Dimitriadis GK, Weickert MO, Randeva HS, Kaltsas G, Grossman A. Medical management of secretory syndromes related to gastroenteropancreatic neuroendocrine tumours. Endocr Relat Cancer. 2016;23(9):R423-36.

25. Khan MS, El-Khouly F, Davies P, Toumpanakis C, Caplin ME. Long-term results of treatment of malignant carcinoid syndrome with prolonged release lanreotide (somatuline autogel). Aliment Pharmacol Ther. 2011;34(2):235-42.

26. Oberg K, Knigge U, Kwekkeboom D, Perren A. Neuroendocrine gastro-entero-pancreatic tumors: ESMO clinical practice guidelines for diagnosis, treatment and follow-up. Ann Oncol. 2012;23(Suppl 7):vii124-30.

27. Strosberg J, Weber J, Feldman M, Goldman J, Almhanna K, Kvols L. Above-label doses of octreotide-LAR in patients with metastatic small intestinal carcinoid tumors. Gastrointest Cancer Res. 2013;6(3):81-5.

28. Strosberg JR, Benson AB, Huynh L, Duh MS, Goldman J, Sahai $\mathrm{V}$, et al. Clinical benefits of above-standard dose of octreotide LAR in patients with neuroendocrine tumors for control of carcinoid syndrome symptoms: a multicenter retrospective chart review study. Oncologist. 2014;19(9):930-6.

29. Weber JM, Feldman M, Kvols L, Strosberg JR. Above-label doses of octreotide-LAR in patients with metastatic small-intestinal carcinoid tumors. J Clin Oncol. 2012;30(15 Suppl):e14579.

30. Riechelmann RP, Pereira AA, Rego JF, Costa FP. Refractory carcinoid syndrome: a review of treatment options. Ther Adv Med Oncol. 2017;9(2):127-37.

31. Toumpanakis C, Garland J, Marelli L, Srirajaskanthan R, Soh J, Davies P, et al. Long-term results of patients with malignant carcinoid syndrome receiving octreotide LAR. Aliment Pharmacol Ther. 2009;30:733-40.

32. Pavel M, Baudin E, Couvelard A, Krenning E, Oberg K, Steinmuller T, et al. ENETS consensus guidelines for the management of patients with liver and other distant metastases from neuroendocrine neoplasms of foregut, midgut, hindgut, and unknown primary. Neuroendocrinology. 2012;95(2):157-76.

33. Lesén E, Björstad $\AA$, Björholt I, Marlow T, Bollano E, Feuilly $\mathrm{M}$, et al. Real-world treatment patterns, resource use and costs of treating uncontrolled carcinoid syndrome and carcinoid heart disease: a retrospective Swedish study. Scand J Gastroenterol. 2018;53(12):1509-18.

34. European Medicines Agency. Xermelo European public assessment report. 2017. https://www.ema.europa.eu/en/documents/ overview/xermelo-epar-summary-public_en.pdf. Accessed 28 Mar 2019.

35. US Food and Drug Administration, Center for Drug Evaluation and Research. Approval package for application number 208794orig1s000 (xermelo). 2017. https://www.accessdata.fda. gov/drugsatfda_docs/nda/2017/208794Orig1s000Approv.pdf. Accessed 28 Mar 2019.

36. Health Canada. Regulatory decision summary - xermelo. 2018. https://hpr-rps.hres.ca/reg-content/regulatory-decision-summarydetail.php?linkID=RDS00469. Accessed 28 Mar 2019.

37. Australian Government Therapeutic Goods Administration. Final scheduling decisions and reasons for decisions by delegates of the secretary to the department of health for matters not referred to an advisory committee (delegate-only decisions). https://www.tga. gov.au/sites/default/files/delegates-final-decisions-jan-2018.pdf. Accessed 30 Apr 2019.

38. Kulke MH, Horsch D, Caplin ME, Anthony LB, Bergsland E, Oberg K, et al. Telotristat ethyl, a tryptophan hydroxylase inhibitor for the treatment of carcinoid syndrome. J Clin Oncol. 2017;35(1):14-23.

39. Cella D, Beaumont JL, Hudgens S, Marteau F, Feuilly M, Houchard A, et al. Relationship between symptoms and health-related quality of life benefits in patients with carcinoid syndrome: Post-hoc analyses from TELESTAR. Clin Ther. 2018;40(12):2006-20.

40. Anthony L, Ervin C, Lapuerta P, Kulke MH, Kunz P, Bergsland $\mathrm{E}$, et al. Understanding the patient experience with carcinoid syndrome: Exit interviews from a randomized, placebo-controlled study of telotristat ethyl. Clin Ther. 2017;39(11):2158-68.

41. Beaumont JL, Cella D, Phan AT, Choi S, Liu Z, Yao JC. Comparison of health-related quality of life in patients with neuroendocrine tumors with quality of life in the general US population. Pancreas. 2012;41(3):461-6.

42. Frojd C, Larsson G, Lampic C, von Essen L. Health related quality of life and psychosocial function among patients with carcinoid tumours A longitudinal, prospective, and comparative study. Health Qual Life Outcomes. 2007;5:18.

43. Swedish Dental and Pharmaceutical Benefits Agency. Xermelo decision for inclusion in drug benefits. https://www.tlv.se/downl oad/18.76aefd0a16385647546422af/1527077011387/bes180517_ xermelo.pdf. Accessed 25 Nov 2019.

44. YolaRx Consultants. Assessment of prevalence and incidence of neuro-endocrine tumors (NETs) and carcinoid syndromes (CS) not adequately controlled by somatostatin analogs in Europe and the United States: Systematic review and target population sizing-final report version 1.0 [Data on file].

45. Korse CM, Taal BG, van Velthuysen ML, Visser O. Incidence and survival of neuroendocrine tumours in the Netherlands according to histological grade: experience of two decades of cancer registry. Eur J Cancer. 2013;49(8):1975-83.

46. Statistics Sweden, 2017. http://www.scb.se/en/. Accessed 21 Mar 2019.

47. Caplin ME, Baudin E, Ferolla P, Filosso P, Garcia-Yuste M, Lim E, et al. Pulmonary neuroendocrine (carcinoid) tumors: European neuroendocrine tumor society expert consensus and recommendations for best practice for typical and atypical pulmonary carcinoids. Ann Oncol. 2015;26(8):1604-20.

48. Pavel M, O'Toole D, Costa F, Capdevila J, Gross D, Kianmanesh $\mathrm{R}$, et al. ENETS consensus guidelines update for the management of distant metastatic disease of intestinal, pancreatic, bronchial neuroendocrine neoplasms (NEN) and NEN of unknown primary site. Neuroendocrinology. 2016;103(2):172-85. 
49. Pavel M, Gross DJ, Benavent M, Perros P, Srirajaskanthan R, Warner RRP, et al. Telotristat ethyl in carcinoid syndrome: safety and efficacy in the TELECAST phase 3 trial. Endocr Relat Cancer. 2018;25(3):309-22.

50. Bhattacharyya S, Toumpanakis C, Chilkunda D, Caplin ME, Davar J. Risk factors for the development and progression of carcinoid heart disease. Am J Cardiol. 2011;107(8):1221-6.

51. Westberg G, Wängberg B, Ahlman H, Bergh CH, BeckmanSuurküla M, Caidahl K. Prediction of prognosis by echocardiography in patients with midgut carcinoid syndrome. Br J Surg. 2001;88(6):865-72.

52. Rubin de Celis Ferrari AC, Glasberg J, Riechelmann RP. Carcinoid syndrome: update on the pathophysiology and treatment. Clinics (Sao Paulo). 2018;73(suppl 1):e490s.

53. Hutchings A, Schey C, Dutton R, Achana F, Antonov K. Estimating the budget impact of orphan drugs in Sweden and France 2013-2020. Orphanet J Rare Dis. 2014;9(1):22.

54. Pollock RF, Meckley LM. An evaluation of the budget impact of a new $20 \%$ subcutaneous immunoglobulin (ig20gly) for the management of primary immunodeficiency diseases in Switzerland. Clinicoecon Outcomes Res. 2018;10:223-9.

55. Woodward TC, Brown R, Sacco P, Zhang J. Budget impact model of tobramycin inhalation solution for treatment of pseudomonas aeruginosa in cystic fibrosis patients. J Med Econ. 2010;13(3):492-9.

56. Huynh L, Totev T, Vekeman F, Neary MP, Duh MS, Benson AB. Cost reduction from resolution/improvement of carcinoid syndrome symptoms following treatment with above-standard dose of octreotide LAR. J Med Econ. 2017;20(9):945-51.

57. Joish VN, Frech F, Lapuerta P. Budgetary impact of telotristat ethyl, a novel treatment for patients with carcinoid syndrome diarrhea: a US health plan perspective. Clin Ther. 2017;39(12):2338-44.

58. Institute for Clinical and Economic Review. ICER value assessment framework. https://icer-review.org/methodology/icers-metho ds/icer-value-assessment-framework/. Accessed 8 May 2019.

59. Cheng MM, Ramsey SD, Devine EB, Garrison LP, Bresnahan BW, Veenstra DL. Systematic review of comparative effectiveness data for oncology orphan drugs. Am J Manag Care. 2012;18(1):47-62.

60. Kanters TA, de Sonneville-Koedoot C, Redekop WK, Hakkaart L. Systematic review of available evidence on 11 high-priced inpatient orphan drugs. Orphanet J Rare Dis. 2013;8:124. 